\title{
SEISMIC RETROFITTING OF DORMITORY BUILDINGS WITH SOFT STOREY AND FLOATING COLUMNS
}

\author{
Nakul Singh ${ }^{1}$, Vikram Singh Kashyap ${ }^{2}$ \\ ${ }^{1}$ M.Tech Scholar, Department of CE, Manipal University Jaipur \\ ${ }^{2}$ Assistant Professor, Department of CE, Manipal University Jaipur
}

\begin{abstract}
Many of the existing building are lacking in adequate earthquake resistance because these are not designed according to modern codes and prevalent earthquake resistant design practice. Also many building that are damaged in earthquake may need not only be repaired but also upgraded of their strength in order to make them seismically resistant. The seismic evaluation and their retrofitting is one of the most challenging task for the structural engineers. The means of retrofitting is to upgrade the strength and structural capacity of the structure to enable it to safely withstand the effect of strong earthquake. There has been a substantial increase in the topic of seismic retrofit of existing buildings in recent years as evidenced by the growing number of research papers published in this area. Attention has been focused worldwide on both building and bridge structures and with the widespread damage to older buildings and bridge structures in the relatively recent bhuj earthquakes, government/owners have begun to take action to prevent similar damage to existing structures in future earthquakes. Open ground storey/soft storey along with the floating columns has been a peculiar feature in the latest multi-storey buildings in India. These features are hugely inadmissible in the buildings which are built in earthquake prone areas. In this Paper, after understanding the behaviour of buildings in earthquakes, why, when, where and how seismic retrofitting is done with shear wall and chevron bracing. Further, a study is performed on an example dormitory building different model with open ground storey and floating columns to highlight the importance of their presence in the seismic analysis using computer program STAAD Pro.V8i The analysis is done with Equivalent Static Analysis/linear elastic static analysis and Response Spectrum Analysis/linear elastic dynamic analysis, as per IS:1893-2002. Various features of lateral stiffness strengthening system, namely lateral bracings, shear walls, increasing the column size in the soft ground storey and their combinations, and are proposed to reduce the stiffness irregularity and discontinuity in the load path incorporated by the soft ground storey and the floating columns respectively. Also, it is inspected that the shear walls are most impressive when used as one long structural wall instead of two short walls having separated by the interrelated beams, and properly placed at the periphery of the buildings to avoid the torsion. It is also noticed that the Chevron braces are most productive when placed under the floating columns to make the force transfer less horizontal
\end{abstract}

Keywords: Floating Column, Dormitory Building, Static And Dynamic Analysis, Seismic Retrofitting, Soft Storey.

\section{INTRODUCTION}

The earthquake at Bhuj, Gujarat, in 2001 has been a watershed event in the earthquake engineering practice in India. The code of practice for seismic analysis, IS 1893:2002 has been revised to reflect the increased seismic demand in many parts of the country. Many existing buildings lack the seismic strength and detailing requirements of IS 1893:2002, IS 4326:1993 and IS 13920: 1993 because they were built prior to the implementation of these codes. This paper Report whose aim is to evolve methodologies to assess the seismic vulnerability of reinforced concrete (RC) G+4 storey dormitory buildings, located in Jaipur urban areas of earthquake zones II and to propose retrofit measures for the structurally deficient buildings.

Casualties caused due to the recent earthquakes have unfolded the vulnerability of the Indian buildings. Most of the engineered constructions, such as multistoried apartments are lacking the very basic features which are required for the resisting earthquakes. This may be because of the lack of awareness of earthquake resistant design and necessary requirements of the Indian codes. Nowadays, especially after the desolating Bhuj earthquake in 2001, there has been a mutual effort throughout India to provide more awareness, especially in practice and education, with respect to earthquake resistant design of structures.

Most of the existing buildings have revealed to deficit observance with the current practice codes, particularly with respect to seismic resistance. This is because of the upgradation of the seismic code (IS: 1893 Part 1-2002). Also the properly designed buildings which were constructed in the past, are lacking the earthquake resistance and the requirements of design codes, such as IS: 4326-1993 and IS: 13920-1993.

The extent of seismic vulnerability can be verified only after a genuine evaluation of the structure is attempted. 
Depending on this evaluation, retrofitting of the vulnerable buildings can be started. The Seismic retrofitting can be done in different ways and to various extents. The purpose should be to certify that the building takes all the damage, but does not collapse when severe earthquake occurs. Seismic retrofitting of a building usually affects the functionality and use during the evaluation and further strengthening. The procedure of believing the users on the importance and necessity for retrofit is also very difficult. Thus, before a project is begun, the aim and procedure of the retrofitting have to be kept in mind.

Earthquakes, the inevitable natural hazards, can cause devastating disasters to our built environment. The catastrophic impact of earthquakes have been "too close for comfort" in the past decade as more moderate to strong earthquakes are striking heavily inhabited regions. These earthquakes, i.e., Northridge in U.S.A. (1994), Kobe in Japan (1995), Golcuk-Izmit in Turkey (1999), JiJi in Taiwan (1999), Gujarat in India (2001) and Nisqually/Seattle in U.S.A. (2001), have caused the loss of thousands of lives and billions in economic loss in the past few years.

Past earthquakes have also illustrated that the failure or collapse of the so-called nonstructural components has caused most casualties and property damage. Quite often, a building sustains only minor structural damage but the building is deemed unsafe to enter or occupy due to extensive damage to its architectural, mechanical and electrical elements and to the building contents after an earthquake. Heavy non-structural in-fill walls, such as brick in-fill walls, are known to potentially change the structural characteristics of a building unfavorably, causing wide spread damage to the building structure.

Recent earthquakes have also demonstrated that these older buildings would have survived, in most cases, with a reasonable upgrading. Satisfactory performance of retrofitted buildings in the latest earthquakes indicates that upgrading older and deficient buildings is the most effective and efficient seismic hazard mitigation measure. In earthquake resistant design, the soft story and the weak story irregularities are reciprocal to a significant difference between the stiffness and the resistance of one of the floors of a building and the rest of them.In this paper, seismic analysis of Dormitory buildings considering structural and geometrical parameters have been carried out using STAAD. Pro software. Soft storeys have been created by increasing the floor heights. Effect of infill has been ignored. Results, in terms of moment, displacement, shear force, axial force and drift are critically examined and salient conclusions are drawn.

\section{STRUCTURAL MODELLING}

AND

\section{ANALYSIS}

\subsection{Material and Geometrical Properties}

Building Type :
(RC) G+4 storey dormitory building

Location

Floors

$:$

floors

Storey height

Foundation depth

Slab

Walls

Reinforced concrete

masonry walls only at periphery.

Live load

Jaipur city

\& terrace floor

Dead load

G.F. + 4 upper

typical floor

Floor finish

Earthquake load

$:$
$:$
$:$

3.5 meter.

1.5 metre

$152.4 \mathrm{~mm}$ thick

$115 \mathrm{~mm}$ thick brick

$3.0 \mathrm{kN} / \mathrm{m} 2$ at typical

$5.19 \mathrm{KN} / m^{2}$ at

1) -2002

Parapet wall load

Concrete density

Brick density

Beam

$1.38 \mathrm{kN} / \mathrm{m} 2$

As per IS-1893 (Part

$4 \mathrm{kn} / \mathrm{m}$

$20 \mathrm{KN} / \mathrm{m}^{3}$

$18.85 \mathrm{KN} / \mathrm{m}^{3}$

Two type of beam

used in this Project work

(1) $450 \times 230 \mathrm{~mm}$

: $\quad$ (2) $900 \times 400 \mathrm{~mm}$ (where floating columns supported on the 1 st storey)

Column

Four type of

columns used which is given as

(1) $400 \times 400 \mathrm{~mm}$

(2) 500x500mm (Column used near middle floating columns for strengthened purpose)

(3) $600 \times 600 \mathrm{~mm}$ (columns used as a braced setback columns in model $6,7,8,9)$

(4) $700 \times 700 \mathrm{~mm}$ (columns used in model $6 \& 7$ braced columns near middle floating columns)

Cantilever Cantilever for floating column from $1^{\text {st }}$ storey $=1.5$ meter

\subsection{Modelling of Building Frames}

Modelling of the building frames are carried out using theSTAAD. Pro software 


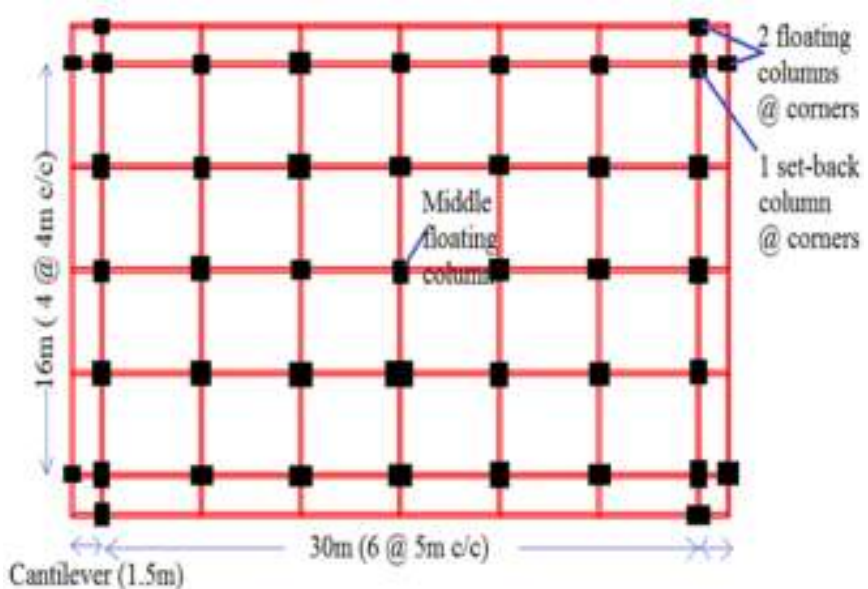

Fig 1: Plan of building

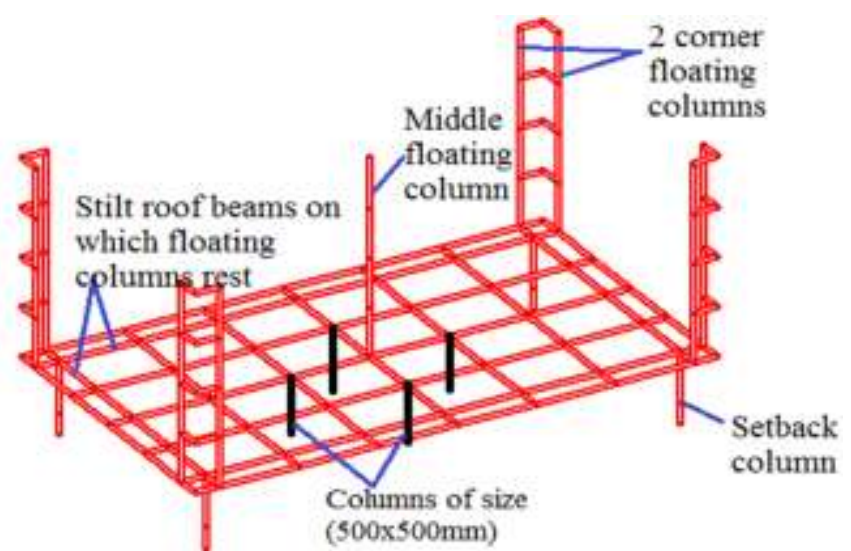

Fig 3: Positioning of floating columns resting on ground roof beams

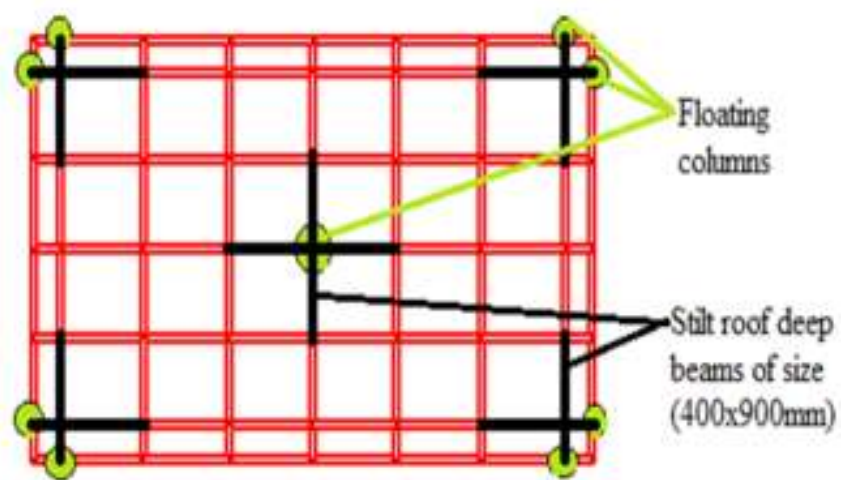

Fig 4:- Beams on which floating columns rest

\subsection{Model of Dormitory Building}

The following 9 cases have been framed for analysis and strengthening purpose of building:-

Model 1: Building has neither shear walls nor lateral bracings in the ground storey-

And brick masonry wall $(115 \mathrm{~mm})$ at periphery of the upper storeys.

Model 2: Building has 4 shear walls (in inner bays) in the ground storey.

Model 3: Building has 4 shear walls (at periphery) in the ground storey.

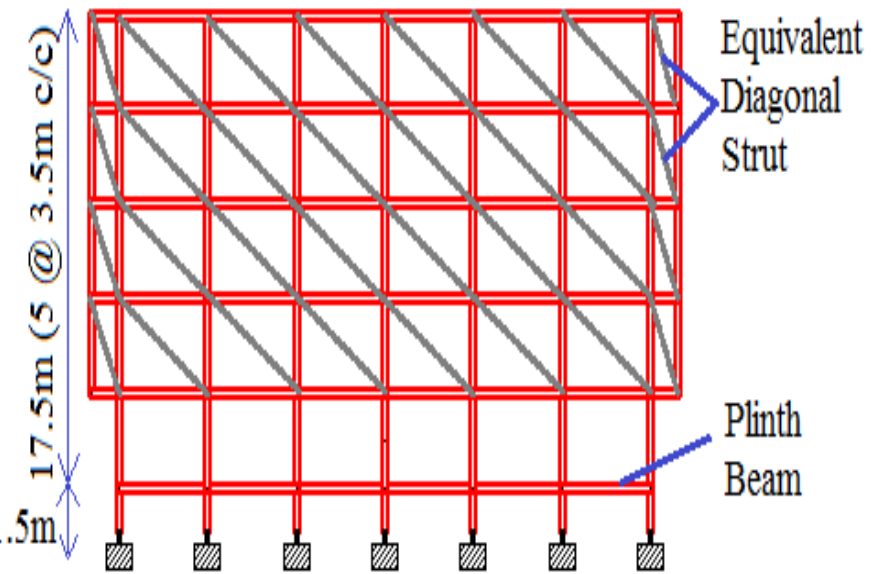

Fig 2: Distribution of height of the building

Model 4: Building has 6 shear walls (1 wall in each strong direction and 2 separate Walls -

In each weak direction) in the ground storey.

Model 5: Building has 4 shear walls (1 wall in each strong direction and 2 combined structural

Walls in each weak direction) in the ground storey.

Model6: Building has concrete bracing (in weak direction only) from the middle floating columnsto the end joints of adjacent vertical columns which have also been strengthened.

Model 7: Building has concrete bracing (in weak direction only) from the middle Floating columnto the mid-span of adjacent vertical strengthened Columns.

Model 8: Combination of model 3 and model 7.

Model 9: Combination of model 5 and model 7. 


\subsection{Simulation of Dormitory Building in STADD.ProV8i}

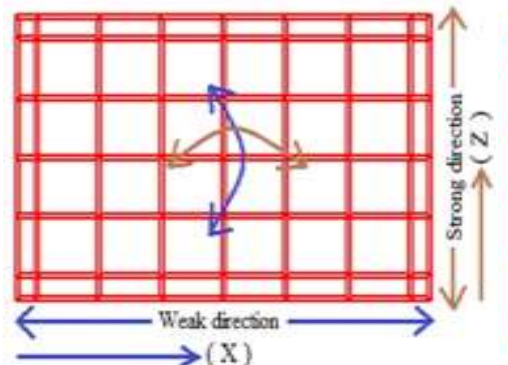

Model No 1 (Plan view)

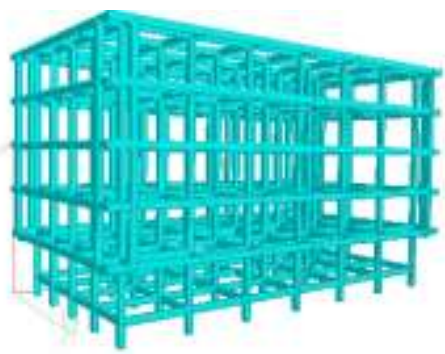

Model No 1 (3-D view)

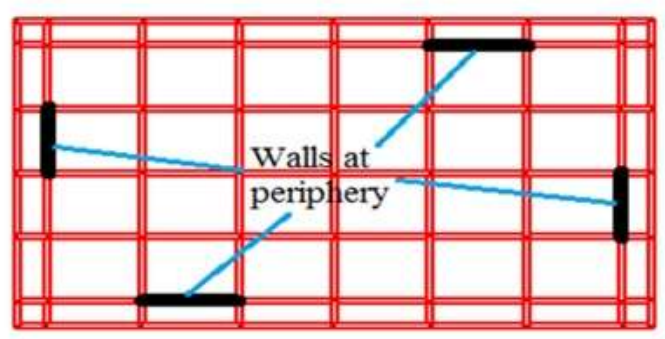

Model No 3 (Plan view)

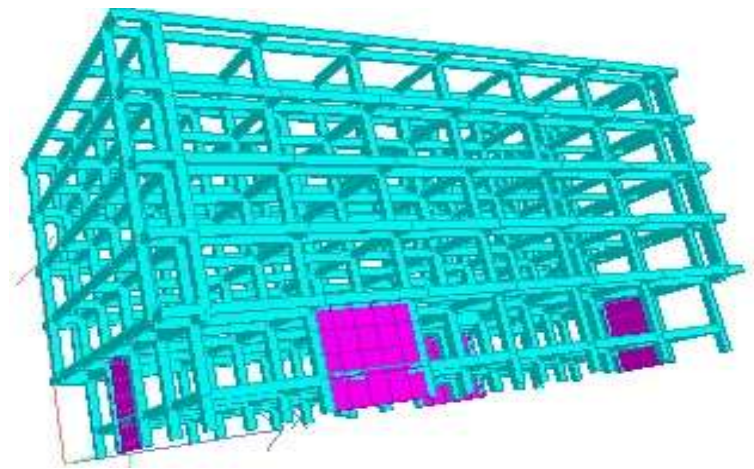

Model No 3 (3-D view)

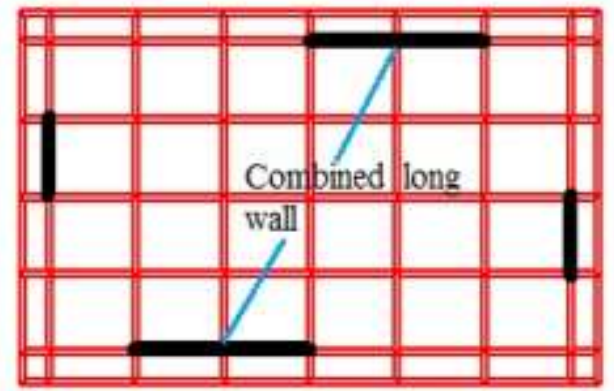

Model No 5 (Plan view)

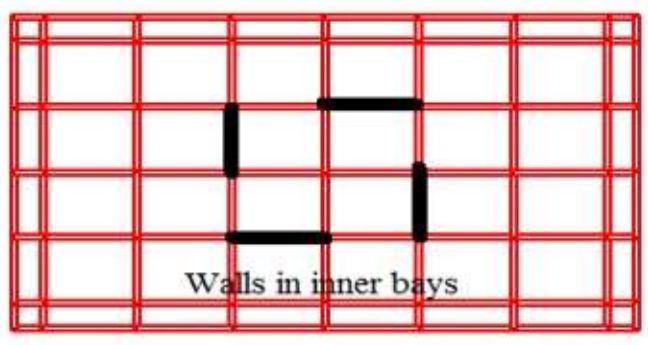

Model No 2 (Plan view)
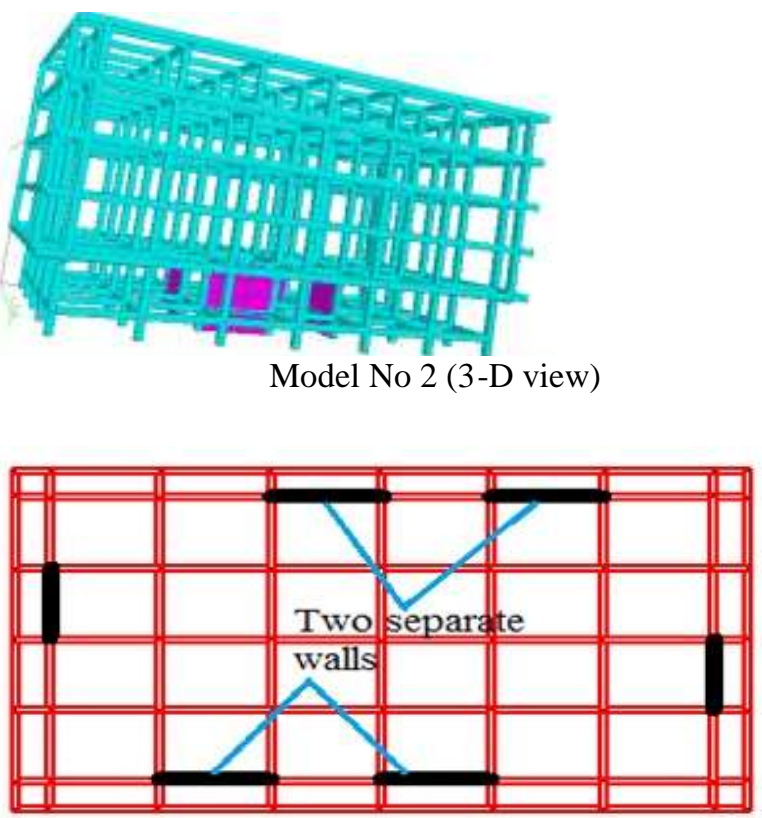

Model No 4 (Plan view)

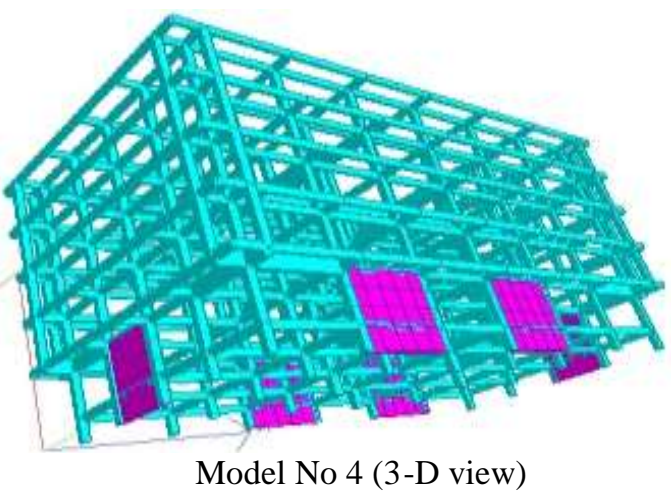

Model No 4 (3-D view)

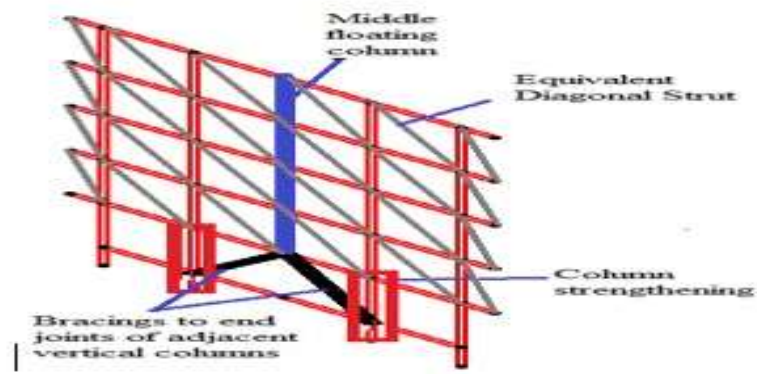

Model No 6 (Bracing view) 


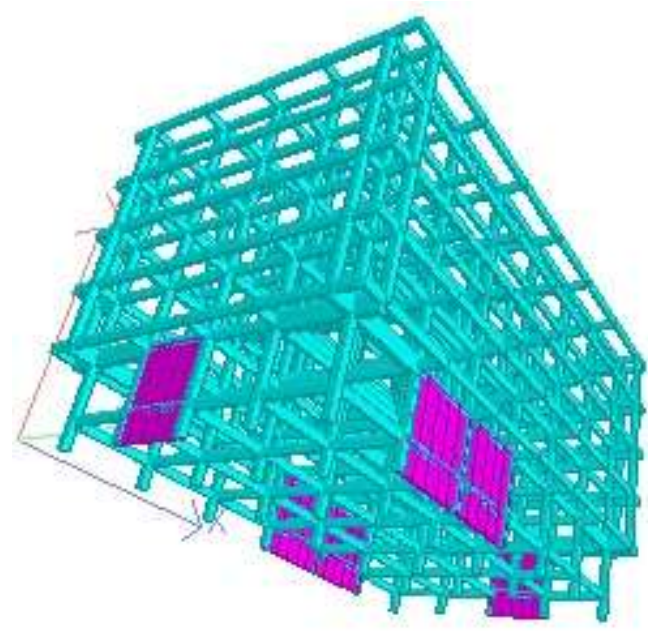

Model No 5 (3-D view)

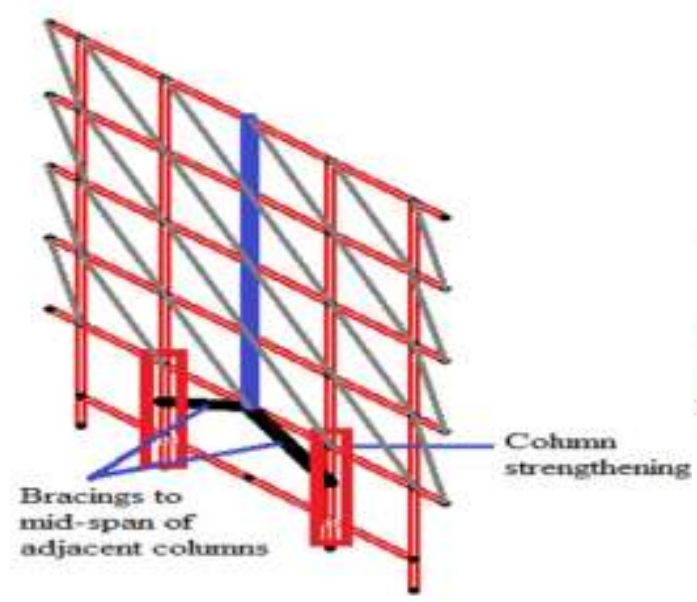

Model No 7 (Bracing view)

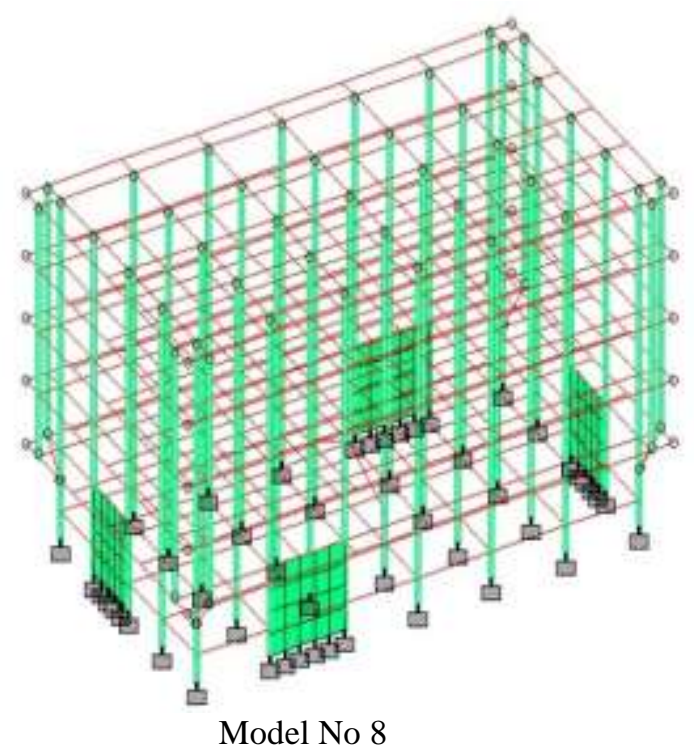

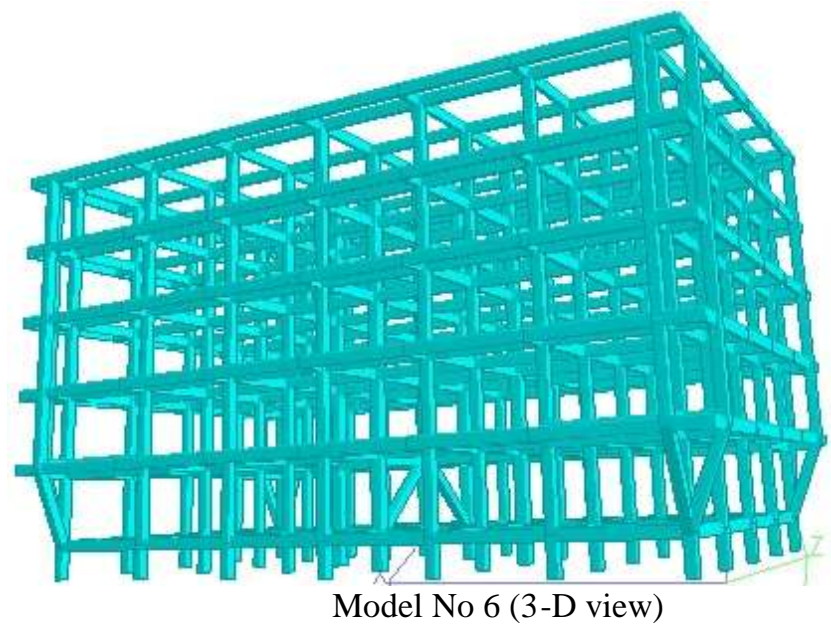

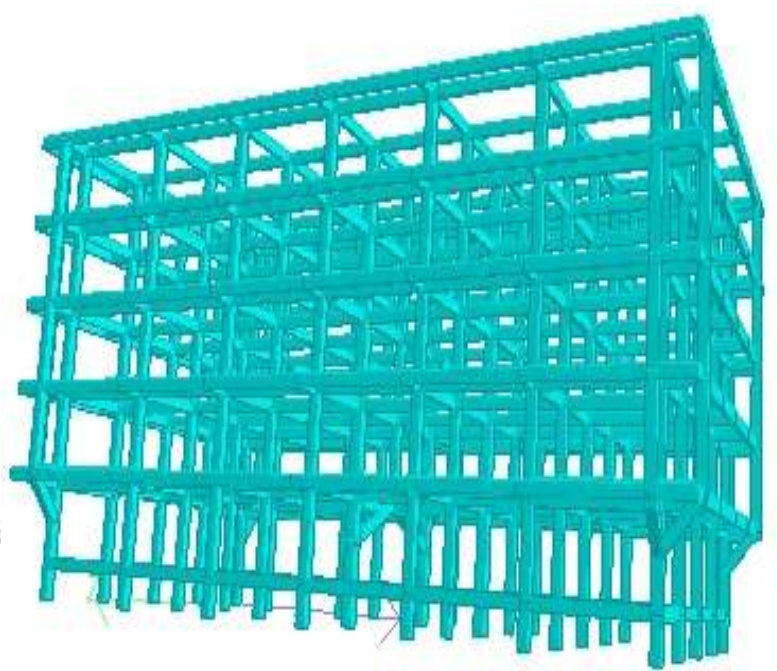

Model No 7 (3-D view)

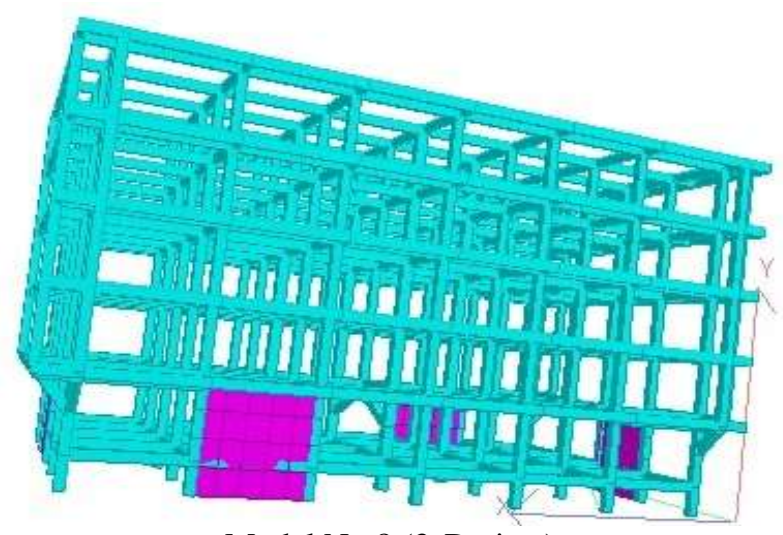

Model No 8 (3-D view) 


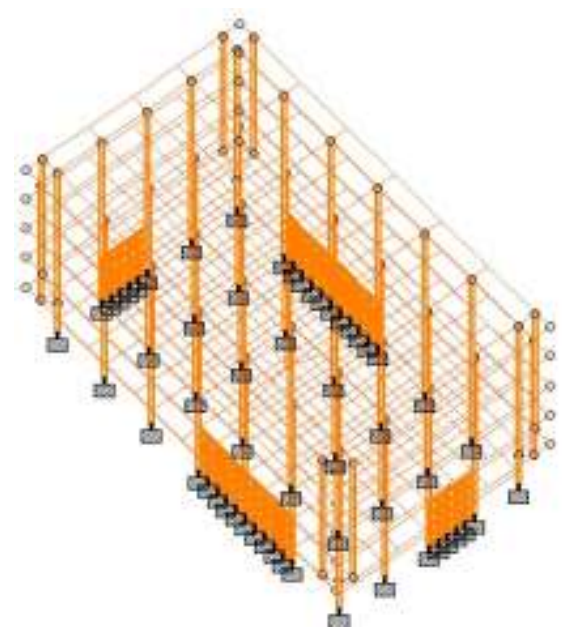

Model No 9

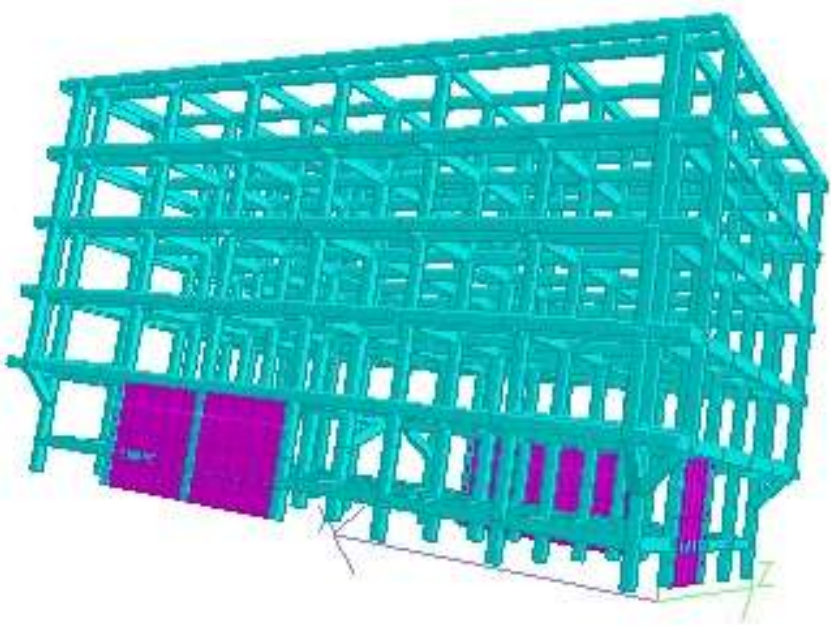

Model No 9 (3-D view)

Fig 5: Different model of dormitoryBuilding (1-9)

\subsection{Loading Conditions}

Typical roof Floor dead load $=5.19 \mathrm{KN} / \mathrm{m}^{2}$

Last roof floor dead load $=30 \%$ reduction of typical floor roof load approx. $3.64 \mathrm{KN} / \mathrm{m}^{2}$

Typical roof Floor load EQL $=5.94 \mathrm{KN} / \mathrm{m}^{2}$

Last roof Floor load $\mathrm{EQL}=4.16 \mathrm{KN} / \mathrm{m}^{2}$

Partition wall used in upper storey $=115 \mathrm{~mm}$

Partition wall load $=6.5 \mathrm{kn} / \mathrm{m}$

Plaster thickness is not taken in calculation

Parapet wall load in upper storey $=4 \mathrm{kn} / \mathrm{m}$

Earthquake loading standard as per IS 1893 part 1:2002

Zone Factor $=0.1$

Response Reduction Factor $=4$

Importance Factor $\quad=1$

Soil Site Factor $\quad=2$

Type of Structure $=1$

Depth of Foundation $=1.5$

Damping $=5 \%$

\subsection{Structural Analysis}

Structural analysis of the building frames are carried out using STAAD.Pro software All the columns are rigidly supported at ground and 20 load combinations, given in Table 1,are considered for the analysis purposes.

Table 1: Details of load cases

\begin{tabular}{|l|l|}
\hline Load Case No. & Load Case Details \\
\hline 1 & $\begin{array}{l}\text { EARTHQUAKE LOAD } \\
\text { IN +X DIRECTION }\end{array}$ \\
\hline 2 & $\begin{array}{l}\text { EARTHQUAKE LOAD } \\
\text { IN +Z DIRECTION }\end{array}$ \\
\hline 3 & $\begin{array}{l}\text { EARTHQUAKE LOAD } \\
\text { IN -X DIRECTION }\end{array}$ \\
\hline 4 & $\begin{array}{l}\text { EARTHQUAKE LOAD } \\
\text { IN +Z DIRECTION }\end{array}$ \\
\hline 5 & DEAD LOAD \\
\hline 6 & LIVE LOAD \\
\hline 7 & $1.5($ DL + LL \\
\hline 8 & $1.2($ DL + LL + EQX) \\
\hline
\end{tabular}

\begin{tabular}{|l|l|}
\hline 9 & $1.2(\mathrm{DL}+\mathrm{LL}-\mathrm{EQX})$ \\
\hline 10 & $1.2(\mathrm{DL}+\mathrm{LL}+\mathrm{EQZ})$ \\
\hline 11 & $1.2(\mathrm{DL}+\mathrm{LL}-\mathrm{EQZ})$ \\
\hline 12 & $1.5(\mathrm{DL}+\mathrm{LL}+\mathrm{EQX})$ \\
\hline 13 & $1.5(\mathrm{DL}+\mathrm{LL}-\mathrm{EQX})$ \\
\hline 14 & $1.5(\mathrm{DL}+\mathrm{LL}+\mathrm{EQZ})$ \\
\hline 15 & $1.5(\mathrm{DL}+\mathrm{LL}-\mathrm{EQZ})$ \\
\hline 16 & $0.9 \mathrm{DL}+1.5 \mathrm{EQ}+\mathrm{X})$ \\
\hline 17 & $0.9 \mathrm{DL}+1.5 \mathrm{EQ}(-\mathrm{X})$ \\
\hline 18 & $0.9 \mathrm{DL}+1.5 \mathrm{EQ}(+\mathrm{Z})$ \\
\hline 19 & $0.9 \mathrm{DL}+1.5 \mathrm{EQ}(-\mathrm{Z})$ \\
\hline 20 & LOAD FOR CHECK \\
\hline
\end{tabular}

\section{RESULTS}

Results of structural analysis can be described under following heads:-

\subsection{Fundamental Natural Period}

The Static (Empirical) and Dynamic (Analytical) fundamental natural periods of the building models have been shown in the Table 2

Table 2: Fundamental Natural Period

\begin{tabular}{|c|c|c|c|c|c|}
\hline \multirow{3}{*}{$\begin{array}{l}\text { Mode } \\
1\end{array}$} & \multicolumn{5}{|c|}{ Fundamental Natural Period (sec) } \\
\hline & \multicolumn{2}{|c|}{ X-Direction } & \multicolumn{2}{|c|}{ Z-Direction } & \multirow{2}{*}{$\begin{array}{l}\text { Torsio } \\
\text { n }\end{array}$} \\
\hline & Static & Dynami & Static & Dynami & \\
\hline 1 & 0.297 & 0.389 & 0.392 & 0.426 & 0.389 \\
\hline 2 & 0.297 & 0.348 & 0.392 & 0.380 & 0.392 \\
\hline 3 & 0.297 & 0.373 & 0.392 & 0.413 & 0.384 \\
\hline 4 & 0.297 & 0.357 & 0.392 & 0.408 & 0.383 \\
\hline 5 & 0.297 & 0.311 & 0.392 & 0.409 & 0.382 \\
\hline 6 & 0.297 & 0.381 & 0.392 & 0.417 & 0.388 \\
\hline 7 & 0.297 & 0.381 & 0.392 & 0.418 & 0.388 \\
\hline 8 & 0.297 & 0.354 & 0.392 & 0.406 & 0.382 \\
\hline 9 & 0.297 & 0.307 & 0.392 & 0.402 & 0.380 \\
\hline
\end{tabular}




\subsection{Lateral Deformation and Base Shear Demand in}

\section{Building}

The lateral displacement profiles of the different models for the two different analyses (Static and Dynamic) have been shown in the below in this figure, the sudden or blunt changes in the slope of the profile reveal the condition of stiffness irregularity. The Seismic Base shear demand in the building are shown in Table 3
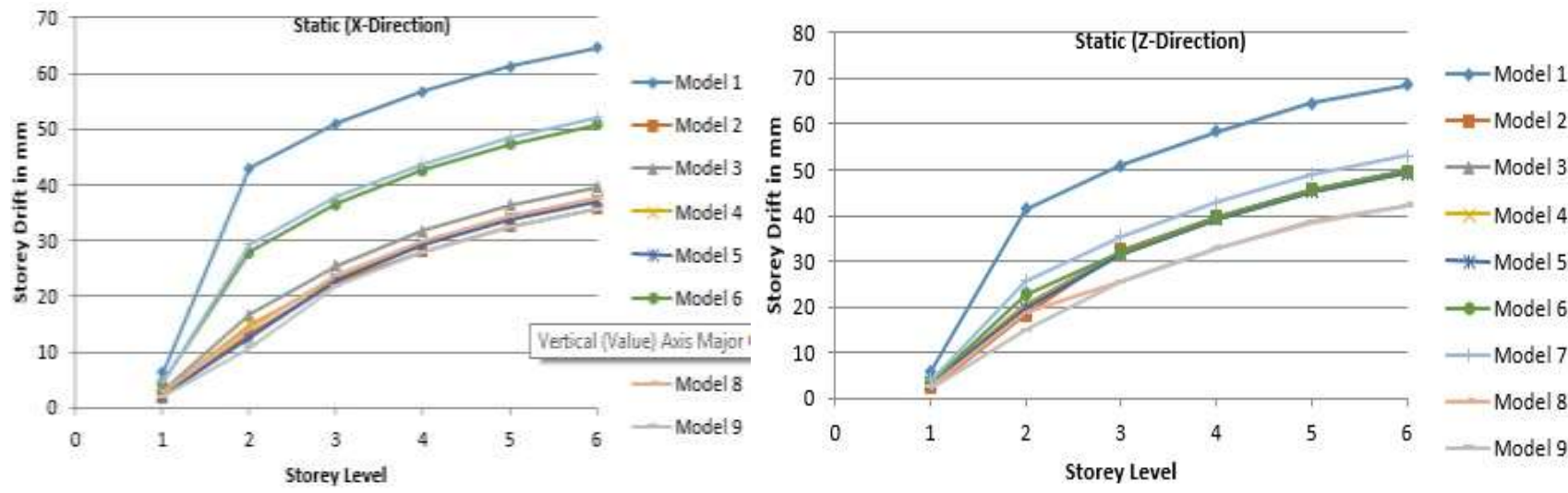

Fig 6: Lateral Displacement Profile by Static Analysis in X and Z direction
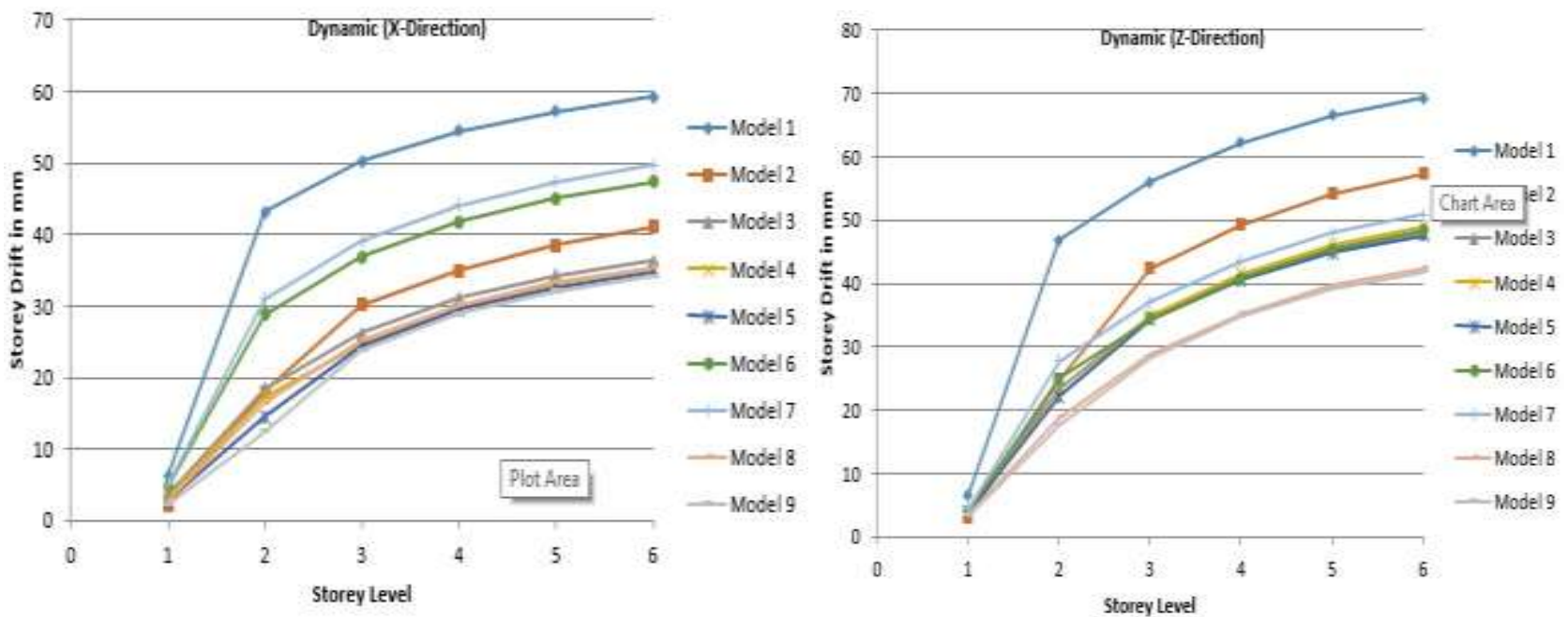

Fig 7: Lateral Displacement Profile by Dynamic Analysis in X and Z direction

Table 3: Seismic Base shear of Building

\begin{tabular}{|c|c|c|c|}
\hline & Seismic Base Shear (KN) & & \\
\hline MODEL & $\mathbf{X}$ & $\mathbf{Y}$ & $\mathbf{Z}$ \\
\hline $\mathbf{1}$ & 1114.58 & 240.17 & 1114.8 \\
\hline $\mathbf{2}$ & 1210.56 & 236.75 & 1185.05 \\
\hline $\mathbf{3}$ & 1288.41 & 223.13 & 1212.95 \\
\hline $\mathbf{4}$ & 1243.81 & 217.05 & 1229.16 \\
\hline $\mathbf{5}$ & 1289.60 & 218.52 & 1231.93 \\
\hline $\mathbf{6}$ & 1202.87 & 206.40 & 1241.87 \\
\hline $\mathbf{7}$ & 1023.00 & 243.06 & 1031.73 \\
\hline $\mathbf{8}$ & 1152.94 & 226.73 & 1066.19 \\
\hline $\mathbf{9}$ & 1111.40 & 222.24 & 1081.08 \\
\hline
\end{tabular}




\subsection{Maximum Moment and Shear in Columns including Set Back Columns}

Table 4: Maximum Moment

\begin{tabular}{|c|c|c|c|c|c|c|c|c|}
\hline \multicolumn{9}{|c|}{ Maximum Moment (kX-w) } \\
\hline \multirow[t]{3}{*}{ Model } & \multicolumn{4}{|c|}{ Static Amalysis } & \multicolumn{4}{|c|}{ Dynamic Analysis } \\
\hline & \multicolumn{2}{|c|}{$\begin{array}{l}\text { Transverse } \\
\text { pirection }\end{array}$} & \multicolumn{2}{|c|}{$\begin{array}{c}\text { Losgitudinal } \\
\text { Direction }\end{array}$} & \multicolumn{2}{|c|}{$\begin{array}{c}\text { Transverse } \\
\text { Direction }\end{array}$} & \multicolumn{2}{|c|}{$\begin{array}{l}\text { Lengitudinal } \\
\text { Direction }\end{array}$} \\
\hline & $\begin{array}{l}\text { Ground } \\
\text { Storey }\end{array}$ & $\begin{array}{l}\text { Upper } \\
\text { Storeys }\end{array}$ & $\begin{array}{l}\text { Ground } \\
\text { Storey }\end{array}$ & $\begin{array}{l}\text { Upper } \\
\text { Storeys }\end{array}$ & $\begin{array}{l}\text { Ground } \\
\text { Storey }\end{array}$ & $\begin{array}{l}\text { Upper } \\
\text { Storeys }\end{array}$ & $\begin{array}{l}\text { Ground } \\
\text { Storey }\end{array}$ & $\begin{array}{l}\text { Epper } \\
\text { Storess }\end{array}$ \\
\hline 1 & 108.299 & 60.29 & 105.912 & 91.05 & 264.764 & 130.520 & 280.12 & 144,963 \\
\hline 2 & 66.654 & 72.546 & 89.885 & 101,867 & 173.973 & 128.283 & 283.822 & 160.981 \\
\hline 3 & 61.813 & 70.023 & 74.821 & 91,416 & 117.724 & 138.619 & 169.623 & 153.194 \\
\hline 4 & 62.734 & 69.576 & 66.898 & 87.627 & 116.478 & 150.162 & 166.844 & 151.390 \\
\hline 5 & 62.419 & 70.935 & 66.868 & 88.559 & 116.133 & 154.436 & 162.695 & 148561 \\
\hline 6 & 170.056 & 93.76 & 148.447 & 100.481 & 333.977 & 191.778 & 325.69 & 192.51 \\
\hline 7 & 149.054 & 97.476 & 162.384 & 109.387 & 296.439 & 156.667 & 290.832 & 155.274 \\
\hline 8 & 358.437 & 222.339 & 266.233 & 168.56 & 43123 & 327.521 & 391.8 & 207.433 \\
\hline 9 & 270.279 & 125.84 & 260365 & 119.822 & $368 .+64$ & 218.717 & 267.355 & 169.149 \\
\hline
\end{tabular}

Table 5: Maximum shear

\begin{tabular}{|c|c|c|c|c|c|c|c|c|}
\hline \multicolumn{9}{|c|}{ Maximum Shear $(\mathrm{KN})$} \\
\hline \multirow[t]{3}{*}{ Medel } & \multicolumn{4}{|c|}{ Static Amalysis } & \multicolumn{4}{|c|}{ Dynamic Analysis } \\
\hline & \multicolumn{2}{|c|}{$\begin{array}{l}\text { Transverse } \\
\text { Direction }\end{array}$} & \multicolumn{2}{|c|}{$\begin{array}{l}\text { Losgitudial } \\
\text { Direction }\end{array}$} & \multicolumn{2}{|c|}{$\begin{array}{c}\text { Transwerse } \\
\text { Direction }\end{array}$} & \multicolumn{2}{|c|}{$\begin{array}{c}\text { L.ongitudial } \\
\text { Direction }\end{array}$} \\
\hline & $\begin{array}{l}\text { Ground } \\
\text { Storey }\end{array}$ & $\begin{array}{l}\text { Tpper } \\
\text { Storeys }\end{array}$ & $\begin{array}{c}\text { Ground } \\
\text { storey }\end{array}$ & $\begin{array}{l}\text { Ipper } \\
\text { Storeys }\end{array}$ & $\begin{array}{l}\text { Ground } \\
\text { Storey }\end{array}$ & $\begin{array}{l}\text { Epper } \\
\text { Storeys }\end{array}$ & $\begin{array}{l}\text { Ground } \\
\text { Storey }\end{array}$ & $\begin{array}{l}\text { Tpper } \\
\text { Storeys }\end{array}$ \\
\hline 1 & 5235 & 48.99 & 55.22 & 3325 & 145.672 & 74989 & 135.848 & 6732 \\
\hline 2 & 46.616 & 54.285 & 33.894 & 36.762 & 107.839 & 87.098 & 89.431 & 75.50 \\
\hline 3 & 38.128 & 48.119 & 22.932 & 35,72 & 93.536 & 81.220 & 61.473 & 71933 \\
\hline 4 & 32.306 & 45.944 & 23.165 & 35.222 & 89.652 & 90.289 & 60.723 & 77.639 \\
\hline 5 & 31.306 & 46.466 & 22.859 & 36160 & 90.199 & 80.697 & 60.998 & 79.116 \\
\hline 6 & 82.212 & 52.423 & 94,595 & 43.54 & 183.036 & $89: 084$ & 186,361 & 85.443 \\
\hline 7 & 171,368 & 52.649 & 165.239 & 42.80 & 246.03 & 73.259 & 246.319 & 69.116 \\
\hline 8 & 211.726 & 82.40 & 241.419 & 98.074 & 327825 & 98.32 & 289.511 & 122.041 \\
\hline 9 & 352.145 & 56.019 & 183.765 & 54.76 & 212.595 & 82,404 & 252.921 & 96,434 \\
\hline
\end{tabular}

\subsection{Maximum Moment and Shear in Braced Set-Back Columns}

Table 6:- Maximum moment and shear

\begin{tabular}{|c|c|c|c|c|c|c|c|c|c|}
\hline \multirow[t]{3}{*}{ Model } & \multirow{3}{*}{$\begin{array}{l}\text { Col.Size } \\
(\mathrm{mm} x \\
\mathrm{mm})\end{array}$} & \multicolumn{4}{|c|}{ Maximum Moment (kN-m) } & \multicolumn{4}{|c|}{ Maximum Shear (kN) } \\
\hline & & \multicolumn{2}{|c|}{$\begin{array}{c}\text { Transverse } \\
\text { Direction }\end{array}$} & \multicolumn{2}{|c|}{$\begin{array}{c}\text { Longitudinal } \\
\text { Direction }\end{array}$} & \multicolumn{2}{|c|}{$\begin{array}{c}\text { Transverse } \\
\text { Direction }\end{array}$} & \multicolumn{2}{|c|}{$\begin{array}{c}\text { Longitudinal } \\
\text { Direction }\end{array}$} \\
\hline & & $\begin{array}{c}\text { Static } \\
\text { Analysis }\end{array}$ & $\begin{array}{l}\text { Dynamic } \\
\text { Analysis }\end{array}$ & $\begin{array}{c}\text { Static } \\
\text { Analysis }\end{array}$ & $\begin{array}{l}\text { Dynamic } \\
\text { Analysis }\end{array}$ & $\begin{array}{c}\text { Static } \\
\text { Analysis }\end{array}$ & $\begin{array}{l}\text { Dynamic } \\
\text { Analysis }\end{array}$ & $\begin{array}{c}\text { Static } \\
\text { Analysis }\end{array}$ & $\begin{array}{l}\text { Dynamic } \\
\text { Analysis }\end{array}$ \\
\hline 1 & 400 & 98.695 & 216.479 & 105.912 & 240.708 & 56.876 & 121.152 & 47.621 & 108.273 \\
\hline 2 & 400 & 62.564 & 139.137 & 97.777 & 244.84 & 46.616 & 123.133 & 29.714 & 69.543 \\
\hline 3 & 400 & 61.813 & 97.643 & 66.946 & 84.541 & 25.523 & 37.049 & 22.679 & 35.743 \\
\hline 4 & 400 & 62.734 & 102.163 & 66.898 & 84.766 & 25.513 & 37.184 & 23.165 & 37.83 \\
\hline 5 & 400 & 62.419 & 102.79 & 66.868 & 84.716 & 25.496 & 37.156 & 28.889 & 37.973 \\
\hline 6 & 600 & 149.03 & 296.977 & 148.447 & 325.69 & 82.212 & 183.036 & 94.594 & 186.361 \\
\hline 7 & 600 & 170.05 & 333.439 & 162.384 & 290.832 & 171.36 & 246.028 & 165.23 & 240.315 \\
\hline 8 & 600 & 358.43 & 310.2 & 266.233 & 284.302 & 211.72 & 264.39 & 241,41 & 273.3 \\
\hline 9 & 600 & 270.27 & 368.40 & 260.365 & 267.355 & 184.89 & 212.595 & 183.76 & 252.921 \\
\hline
\end{tabular}

\subsection{Maximum Moment and Shear in Columns around Middle Floating Column}

Table 7: Maximum Moment and Shear in columns around middle floating column

\begin{tabular}{|c|c|c|c|c|c|c|c|}
\hline \multirow[t]{3}{*}{ Model } & \multirow{3}{*}{$\begin{array}{c}\text { Col. } \\
\text { Size } \\
(\mathbf{m m} \\
\mathbf{x} \\
\mathbf{m m})\end{array}$} & \multicolumn{2}{|c|}{ Maximum Moment (kN-m) } & \multicolumn{4}{|c|}{ Maximum Shear (kN) } \\
\hline & & \multicolumn{2}{|c|}{ Transverse Direction $\left(\mathrm{M}_{3}\right)$} & \multicolumn{2}{|c|}{$\begin{array}{c}\text { Transverse Direction } \\
\text { (F) }\end{array}$} & \multicolumn{2}{|c|}{$\begin{array}{l}\text { Longitudinal } \\
\text { Direction }\left(F_{s}\right)\end{array}$} \\
\hline & & $\begin{array}{c}\text { Static } \\
\text { Analyxiz }\end{array}$ & Dynamic Analysix & $\begin{array}{c}\text { Static } \\
\text { Analysis }\end{array}$ & $\begin{array}{l}\text { Dynamic } \\
\text { Analyzix }\end{array}$ & $\begin{array}{c}\text { Static } \\
\text { Analyzis }\end{array}$ & $\begin{array}{l}\text { Dynamic } \\
\text { Analysix }\end{array}$ \\
\hline 1 & 500 & 276.662 & 494.093 & 144.791 & 265.023 & 126.572 & 238.398 \\
\hline 2 & 500 & 245.886 & 455.319 & 119.305 & 202.254 & 99.839 & 205.858 \\
\hline 3 & 500 & 250.726 & 423.126 & 137.252 & 279.057 & 104.024 & 197.682 \\
\hline 4 & 500 & 248.601 & 409.248 & 135.057 & 268.005 & 103.431 & 196.144 \\
\hline 5 & 500 & 249.194 & 423.247 & 135.191 & 268.548 & 103.686 & 196.736 \\
\hline 6 & 700 & 287.013 & 599.426 & 140.34 & 283.472 & 131.94 & 312.507 \\
\hline 7 & 700 & 359.636 & 566.29 & 429.019 & 562.693 & 158.085 & 293.793 \\
\hline
\end{tabular}




\subsection{Shear Force in Beams on which Floating Columns Rest}

Table 8: Maximum Shear in beams on which floating columns rest

\begin{tabular}{|c|c|c|c|c|c|c|c|c|}
\hline \multirow[t]{4}{*}{ Model } & \multicolumn{8}{|c|}{ Maximum Shear (kN) } \\
\hline & \multicolumn{4}{|c|}{ Intermediate Beam } & \multicolumn{4}{|c|}{ Overhang Beam } \\
\hline & \multicolumn{2}{|c|}{$\begin{array}{c}\text { Transverse } \\
\text { Direction }\left(F_{x}\right)\end{array}$} & \multicolumn{2}{|c|}{$\begin{array}{l}\text { Longitudinal } \\
\text { Direction }\left(F_{y}\right)\end{array}$} & \multicolumn{2}{|c|}{$\begin{array}{c}\text { Transverse } \\
\text { Direction }\left(\mathbf{F}_{\boldsymbol{z}}\right)\end{array}$} & \multicolumn{2}{|c|}{$\begin{array}{l}\text { Longitudinal } \\
\text { Direction }\left(\mathbf{F}_{y}\right)\end{array}$} \\
\hline & $\begin{array}{c}\text { Static } \\
\text { Analysis }\end{array}$ & $\begin{array}{l}\text { Dynamic } \\
\text { Analysis }\end{array}$ & $\begin{array}{c}\text { Static } \\
\text { Analysis }\end{array}$ & $\begin{array}{l}\text { Dynamic } \\
\text { Analysis }\end{array}$ & $\begin{array}{c}\text { Static } \\
\text { Analysis }\end{array}$ & $\begin{array}{l}\text { Dynamic } \\
\text { Analysis }\end{array}$ & $\begin{array}{c}\text { Static } \\
\text { Analysis }\end{array}$ & $\begin{array}{l}\text { Dynamic } \\
\text { Analysis }\end{array}$ \\
\hline 1 & 12.91 & 8.842 & 447.901 & 536.147 & 13.014 & 32.18 & 396.427 & 503.519 \\
\hline 2 & 29.252 & 73.656 & 452.637 & 461.987 & 32.681 & 87.914 & 391.353 & 519.508 \\
\hline 3 & 32.137 & 81.225 & 444.99 & 549.594 & 42.168 & 96.033 & 410.975 & 555.978 \\
\hline 4 & 30.757 & 78.244 & 444.841 & 543.802 & 32.713 & 75.179 & 411.471 & 558.09 \\
\hline 5 & 32.463 & 81.678 & 447.785 & 544.022 & 38.845 & 83.212 & 410.505 & 557.044 \\
\hline 6 & 20.747 & 55.73 & 226.891 & 432.213 & 30.572 & 45.731 & 155.243 & 286.905 \\
\hline 7 & 14.175 & 35.592 & 315.982 & 379.661 & 27.088 & 29.967 & 206.881 & 286.191 \\
\hline
\end{tabular}

\section{DISCUSSION}

\subsection{Model 1 versus Model 2}

Introduction of shear walls in the soft first storey have helped in decreasing the displacement of building globally, as these walls are like plates of the structural elements which have huge in plane strength and stiffness. They resist the lateral forces through united action of flexure, axial, and

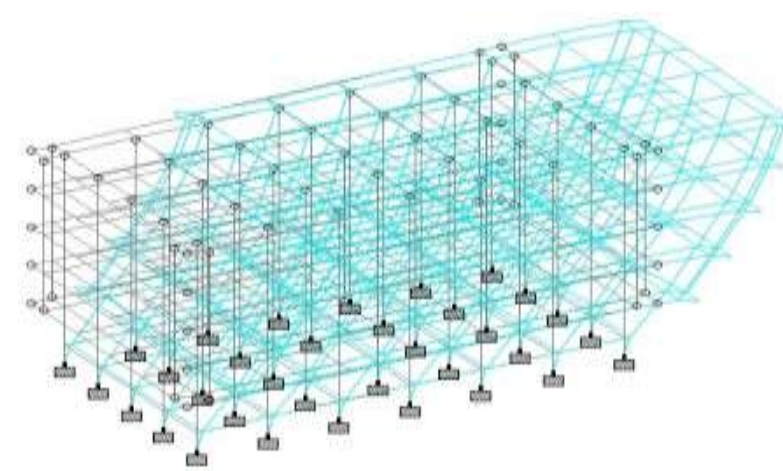

Fig 8: Translation in Model 1 shear. These shear walls, because of their huge initial lateral stiffness, helps in reducing the shear forces and the bending moments in the beams and columns when provided along with the reinforced concrete moment resisting frames and by being the most crucial part of lateral load resisting system. They have also indirectly succeeded in reducing the stiffness irregularity in the building.

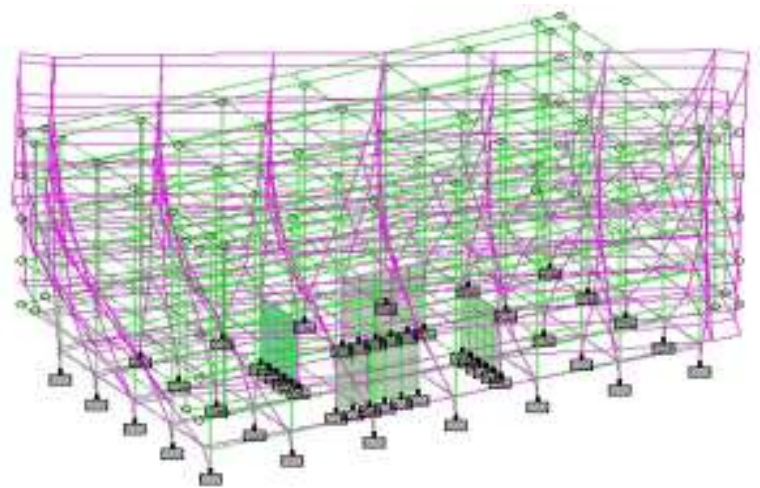

Fig 9: Translation in Model 2

\subsection{Model 2 versus Model 3}

The Building Models 2 and 3 have the same number and the size of the shear walls but at separate locations such as, shear walls in the inner bays and at the periphery of the building respectively. Generally, by keeping the same shear walls nearing the centre of the building which indirectly leads to the tendency of the building to undergo torsion. This unwanted torsion probably occurred because of an eccentricity between the Centre of mass and Centre of rigidity. Hence, the shear walls are the most impressive when usually kept at the periphery of the buildings. 


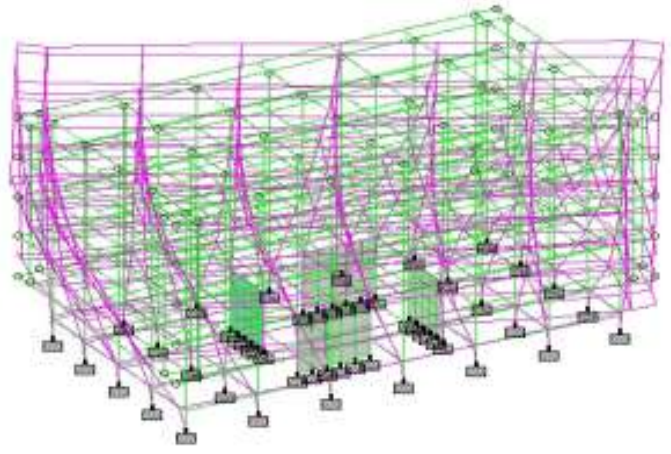

Fig 10: Translation in Model 2

\subsection{Model 4 versus Model 5}

The Building Models 4 and 5, have same the size of the shear walls but at separate locations in the X-direction at the periphery such as, two short shear walls and one combined long shear wall respectively. Since, the concerned wall area is nonetheless the same in both the buildings, but the building with longer shear wall is more unyielding than the others, and thus, allows the building to be more resist

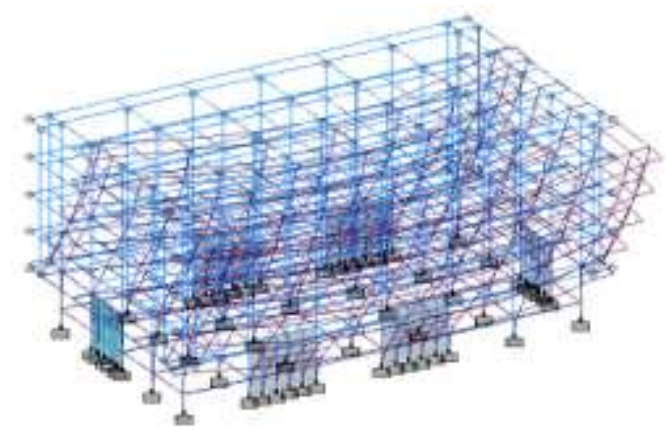

Fig 12: Translation in Model 4

\subsection{Model 1 versus Model 6}

Introduction of the Chevron bracings, in the soft first storey, have greatly helped in globally reducing the lateral deformation of building. In Model 1, without the braces, the transfer of forces is not vertical, which has led the beams (on which the floating columns rest) carry the forces horizontally to the continuous columns, and get heavily stressed in shear. But after the ingression of the chevron braces in the Model 6, the transfer of forces has become not only more vertical, but also not more horizontal.

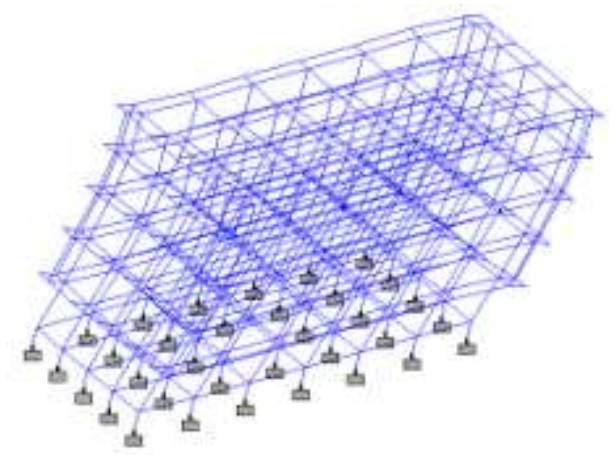

Fig 14: Model 1 (SHEAR ACTION)

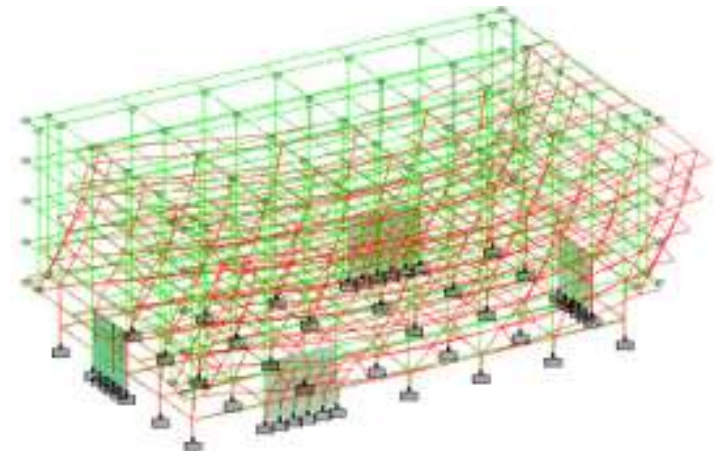

Fig 11: Translation in Model 3

against the lateral motion, and hence, the lateral deformation and the shear force and bending moment demands on the beams and columns have been tremendously reduced by properly using the double length shear walls.

Therefore, it is beneficial to keep one long shear wall instead of the two short walls having separated by the interrelated beams.

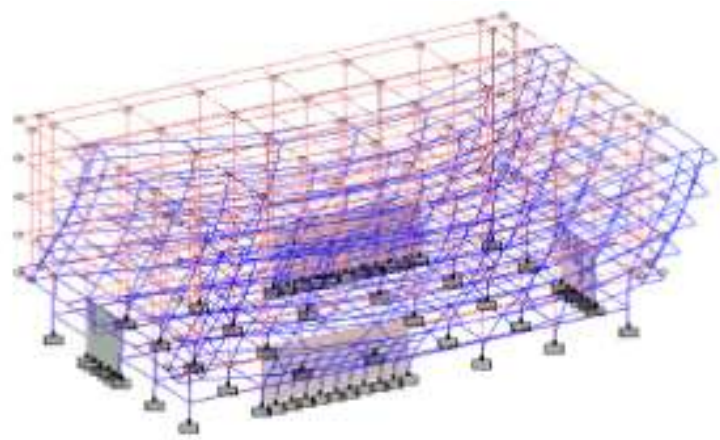

Fig 13: Translation in Model 5

The forces have been transferred to the beams and the columns through the brace, thereby relieving the girder beams, hence, the bracings have indirectly reduced the discontinuity in the load path of the vertical forces in a building. Therefore, for a soft first storey, the bracings should be kept in relevant bays so as to hold the functional use of the open storey.

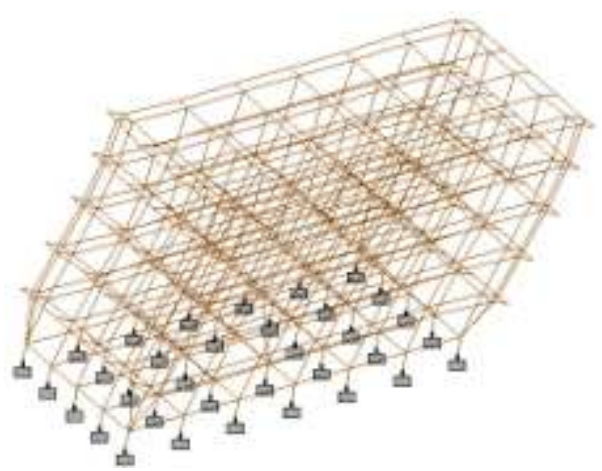

Fig 15: Model 6 (AXIAL ACTION) 


\subsection{Model 6 versus Model 7}

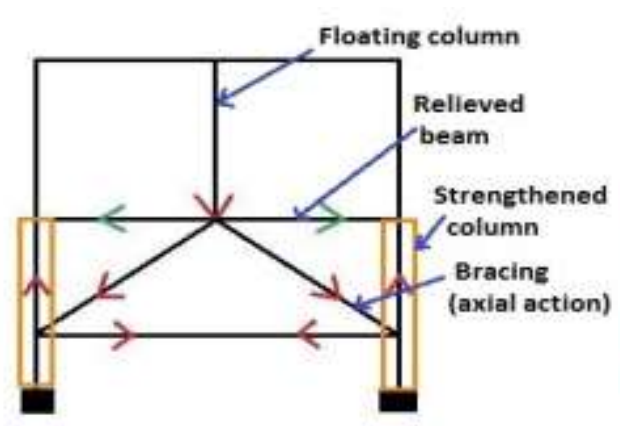

Fig 16: Model 6(AXIAL ACTION)

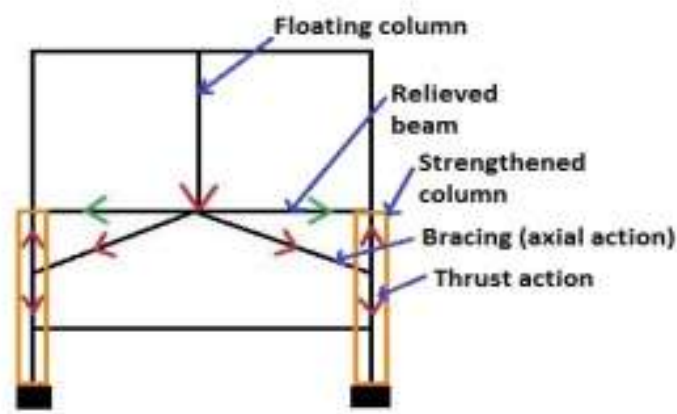

Fig 17: Model 7 (AXIAL+THRUST ACTION)

Even though, the lateral deformation of the building in model 7 increase considerable, and the bending moments and the shear forces of the open ground storey columns, are less in Model 6, Model 7 is preferred for the movement and accommodation of cars for parking in the open ground storey.

\subsection{Model 8 versus Model 9}

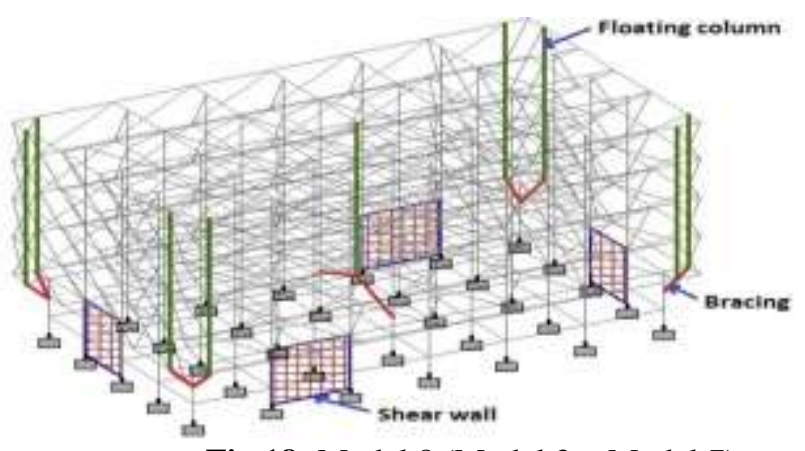

Fig 18: Model 8 (Model $3+$ Model 7)

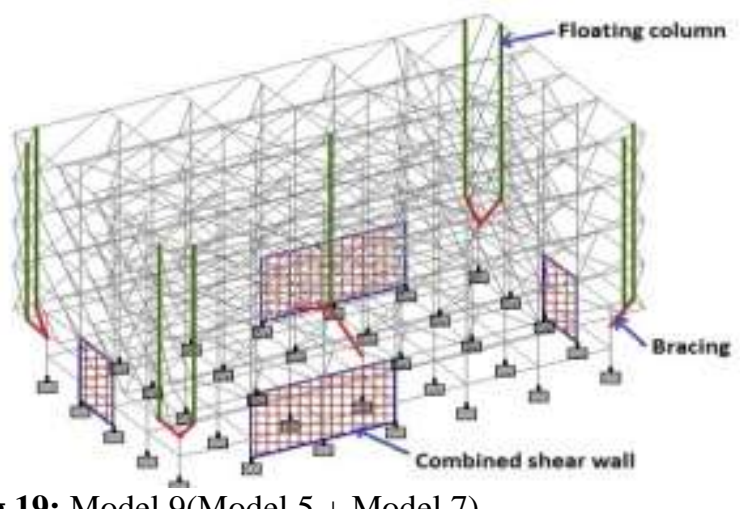

Fig 19: Model 9(Model $5+$ Model 7)

Both the Models 8 and 9 are combination of all the lateral strengthening techniques discussed. Both the lateral strengthening techniques, i.e. the structural or shear walls and the Chevron braces along with column strengthening, are incorporated in the building to get the best structural configuration in terms of seismic resistance.

For severe parking requirements, Model 8 can be preferred, where a single structural wall in each direction is placed, along with the braces, And for moderate parking requirements, Model 9 can be preferred, where a long combined structural wall in a particular direction is placed, along with the braces.

\section{CONCLUSION}

The conclusions can be enumerated point wise as follows:-

1. When Model 1 and Model 2 are compared, Model 2 is preferred because of the incorporation of the shear walls in the opens storey, thereby helps in minimizing

the lateral deformationand the stiffness irregularity of building.

2. When Model 2 and Model 3 are compared, Model 3 is preferred because of the tendency of Model 2 to go under torsion which occurs because of an eccentricity between the centre of mass and the centre of rigidity. Hence, the shear walls are the most impressive when usually kept at the periphery of the buildings.

3. When Model 4 and Model 5 are compared, Model 5 is preferred because the building with the longer shear wall is much stronger than the others, and therefore, allows the building to be more defiant towards the lateral motion, and thereby, the lateral deformation and force demands on beams and columns are extremely reduced by using the double length shear walls. Therefore, it is salubrious to keep one long shear wall instead of the two short walls having separated by the interrelated beams.

4. When Model 1 and Model 6 are compared, Model 6 is preferred because of the incorporation of the Chevron 
bracings in the soft ground storey which allows the vertical forces to get transferred through the combined action of shear and thrust, former for the beam and latter for the column, thereby alleviating the beams and hence, mitigating the discontinuity in the load path of the building. Therefore, for a soft first storey, the bracings should be kept in relevant bays so as to hold the functional use of the open storey.

5. When Model 6 and Model 7 are compared, Model 7 is preferred for the movement and accommodation of cars for parking in the open ground storey.

6. When Model 8 and Model 9 are compared, the preference depends on the severity of parking requirements.

For severe parking requirements, Model 8 can be preferred, where a single structural wall in each direction is placed, along with the braces.

For moderate parking requirements, Model 9 can be preferred, where a long combined structural wall in a particular direction is placed, along with the braces.

\section{REFERENCES}

[1]. ATC 40 (1996), [The Seismic Evaluation and Retrofit of Concrete Buildings: Vol. 1], Applied Technology Council, USA.

[2]. IS; 456-2000, [Plain and Reinforced Concrete - Code of Practice], Bureau of Indian Standards.

[3]. IS: 800 (Draft), [The Indian Standard Code of Practice for General Construction in Steel], Bureau of Indian Standards.

[4]. IS: 1893-2002, [The Criteria for Earthquake Resistant Design of Structures], Part 1: General Provisions and Buildings, Bureau of Indian Standards.

[5]. IS: 4326-1993, [The Earthquake Resistant Design and Construction of Buildings - Code of Practice], Bureau of Indian Standards.

[6]. IS: 13920-1993, [The Indian Standard Code of Practice for Ductile Detailing of Reinforced Concrete Structures Subjected to Seismic Forces], Bureau of Indian Standards.

[7]. IS: 13935-1993, [The Indian Standard for Repair and Seismic Strengthening of Buildings - Guidelines], Bureau of Indian Standards.

[8]. Basu, P. C. (2002), [The Seismic Up gradation of Buildings: An Overview], the Indian Concrete Journal, the Associated Cement Companies Ltd., August, pp. 471-485.

[9]. IS: 13827-1993, [Improving the Earthquake Resistance of Earthen Buildings], Bureau of Indian Standards, New Delhi.

[10]. [IITK-GSDMA Guidelines for Seismic Evaluation and Strengthening of Buildings], (2005), Indian Institute of Technology Kanpur.

[11]. Manual for Seismic Evaluation and Retrofit of Multistoried RC Buildings], (2005), Indian Institute of Technology Madras and Structural Engineering Research Centre, Chennai. Project sponsored by Department of Science and Technology, Government of India.
[12]. Draft Indian Standard on, [Seismic Evaluation and Strengthening of Existing Reinforced Concrete Buildings] Guidelines, Bureau of Indian Standards.

[13]. Agarwal, P. and Shrikhande, M. (2005), [The Earthquake Resistant Design of Structures], Prentice-Hall of India Pvt. Ltd. 